\title{
A momentum-dependent perspective on quasiparticle interference in $\mathrm{Bi}_{2} \mathrm{Sr}_{2} \mathrm{CaCu}_{2} \mathrm{O}_{8+\delta}$
}

\author{
I. M. Vishik ${ }^{1,2}$, E. A. Nowadnick ${ }^{1,2}$, W. S. Lee ${ }^{1,2}$, Z. X. Shen ${ }^{1,2}$, B. Moritz ${ }^{1}$, T. P. Devereaux ${ }^{1 \star}$, K. Tanaka ${ }^{3}$, \\ T. Sasagawa ${ }^{4}$ and T. Fujiii ${ }^{5}$
}

\begin{abstract}
Angle-resolved photoemission spectroscopy (ARPES) probes the momentum-space electronic structure of materials and provides invaluable information about the high-temperature superconducting cuprates'. Likewise, scanning tunnelling spectroscopy (STS) reveals the cuprates' real-space inhomogeneous electronic structure. Recently, researchers using STS have exploited quasiparticle interference (QPI)-wave-like electrons that scatter off impurities to produce periodic interference patterns-to infer properties of the quasiparticles in momentum space. Surprisingly, some interference peaks in $\mathrm{Bi}_{2} \mathrm{Sr}_{2} \mathrm{CaCu}_{2} \mathrm{O}_{8+\delta}$ (Bi-2212) are absent beyond the antiferromagnetic zone boundary, implying the dominance of a particular scattering process ${ }^{2}$. Here, we show that ARPES detects no evidence of quasiparticle extinction: quasiparticle-like peaks are measured everywhere on the Fermi surface, evolving smoothly across the antiferromagnetic zone boundary. This apparent contradiction stems from differences in the nature of single-particle (ARPES) and two-particle (STS) processes underlying these probes. Using a simple model, we demonstrate extinction of QPI without implying the loss of quasiparticles beyond the antiferromagnetic zone boundary.
\end{abstract}

Recently, STS has been used to infer momentum-space information from the Fourier transform of the position $(\mathbf{r})$ - and energy $(\omega)$-dependent local density of states (LDOS), $\rho(\mathbf{r}, \omega)$ (refs 3-6). Conventionally, a superconductor has well-defined momentum eigenstates (that is, Bogoliubov quasiparticles), so $\rho(\mathbf{r}, \omega)$ is spatially homogeneous. However, scattering from inhomogeneities often present in the cuprates ${ }^{7}$ mixes momentum eigenstates and QPI manifests itself as a spatial modulation of $\rho(\mathbf{r}, \omega)$ with well-defined wave vector $\mathbf{q}$, appearing in the Fourier transform, $\rho(\mathbf{q}, \omega)$. QPI experiments are interpreted using the octet $\operatorname{mode}^{3-6}$, positing that wave vectors $\mathbf{q}_{1-7}$ connecting the ends of 'banana shaped' contours of constant energy (CCEs) dominate $\rho(\mathbf{q}, \omega)$. Dispersing wave vectors are associated with coherent superconducting quasiparticles and the evolution of $\mathbf{q}_{1-7}$ as a function of bias voltage is used to infer the Fermi surface and the magnitude of the $d$-wave superconducting gap. QPI experiments have found that the intensity of some of the peaks in $\rho(\mathbf{q}, \omega)$ vanishes on approaching the diagonal line between $(0, \pi)$ and $(\pi, 0)$ (corresponding to the antiferromagnetic zone boundary $)^{5}$. These results lead naturally to speculation about the disappearance of QPI and possible extinction of quasiparticles themselves near the boundary of the Brillouin zone (antinodal region).

Notably, ARPES has long shown antinodal quasiparticles below the critical temperature $T_{\mathrm{c}}$ in $\mathrm{Bi}-2212$ over a wide doping range
( $p>0.08$; refs 8-10), although this point has not been a focus in the literature. In Fig. 1, we report data on single crystals of Bi-2212 at four dopings spanning much of the same doping range as reported in recent STS studies ${ }^{5}$, from slightly underdoped $\left(T_{\mathrm{c}}=92 \mathrm{~K}\right.$, denoted UD92) to substantially underdoped (UD50). ARPES measurements were carried out at Beamline 5-4 of the Stanford Synchrotron Radiation Lightsource using a Scienta R4000 electron analyser. Samples were cleaved in situ at a pressure better than $5 \times 10^{-11}$ torr and measured at $10 \mathrm{~K}$ with an energy resolution of $8 \mathrm{meV}$. UD50 was measured with $19-\mathrm{eV}$ photons with cuts parallel to $\Gamma Y$ and the other samples were measured with $22.7-\mathrm{eV}$ photons with cuts parallel to $\Gamma M$. These data demonstrate sharp peaks at low binding energy, associated with coherent quasiparticles, in all of the energy distribution curves (EDCs) around the Fermi surface, strongly arguing against extinction in the single-particle spectrum. On the other hand, Fourier-transform STS studies report dispersing QPI over only a limited energy range, corresponding to the region of the Fermi surface intermediate between the node and the antinode. In this energy range, both ARPES and Fourier-transform STS show a similar Fermi surface for samples with $p \sim 11 \%$ (Fig. 1f), but quasiparticles are clear in ARPES both in the nodal region and in the antinodal region, the latter being the focus of our study. Although the antinode is associated with pseudogap physics, antinodal quasiparticles seem to 'know' about superconductivity: for underdoped Bi-2212, the antinodal quasiparticle weight has been shown to scale with the superfluid density ${ }^{2,8}$ and $T_{\mathrm{c}}$. The inset of Fig. If illustrates that the quasiparticle peak emerges near the antinode only below $T_{\mathrm{c}}$ (ref. 11), whereas the antinodal gap seems to smoothly evolve into the pseudogap above $T_{\mathrm{c}}$.

The EDCs in Fig. 1 were fitted to a simple model to assess whether there is anomalous scattering near the antiferromagnetic zone boundary. Although many factors contribute to the amplitude and width of the quasiparticle peaks, including matrix element and $k_{z}$ effects ${ }^{12}$, qualitative conclusions about the single-particle scattering rate can be deduced by analysing the momentum dependence of the peak width. Symmetrized EDCs were fitted to a spectral function with an energy-dependent scattering rate, $\Gamma(\omega)=\alpha \omega$, convolved with the experimental energy resolution. A similar model was found to provide a robust fit for the spatially inhomogeneous STS conductances seen in Bi-2212 (ref. 13). At $\mathbf{k}=\mathbf{k}_{\mathrm{F}}$, the only free parameters are the prefactor, $\alpha$, and the gap energy, $\Delta(\mathbf{k})$; together, these define a characteristic scattering rate, $\Gamma_{2}^{*}(\mathbf{k})=\alpha \Delta(\mathbf{k})$. Figure 2 shows $\Gamma_{2}^{*}$ as a function of Fermi surface angle for UD75 and UD92, plotted together with the fitted gap. Near the antinode, the peak width is smaller than the gap, implying that

\footnotetext{
${ }^{1}$ Stanford Institute for Materials and Energy Sciences, SLAC National Accelerator Laboratory, 2575 Sand Hill Road, Menlo Park, California 94025, USA, ${ }^{2}$ Geballe Laboratory for Advanced Materials, Departments of Physics and Applied Physics, Stanford University, California 94305, USA, ${ }^{3}$ Department of Physics, Osaka University, Osaka, 560-0043, Japan, ${ }^{4}$ Materials and Structures Laboratory, Tokyo Institute of Technology, Yokohama-City, 226-8503, Japan, ${ }^{5}$ Cryogenic Center, University of Tokyo, Tokyo, 113-0032, Japan. *e-mail: tpd@stanford.edu.
} 

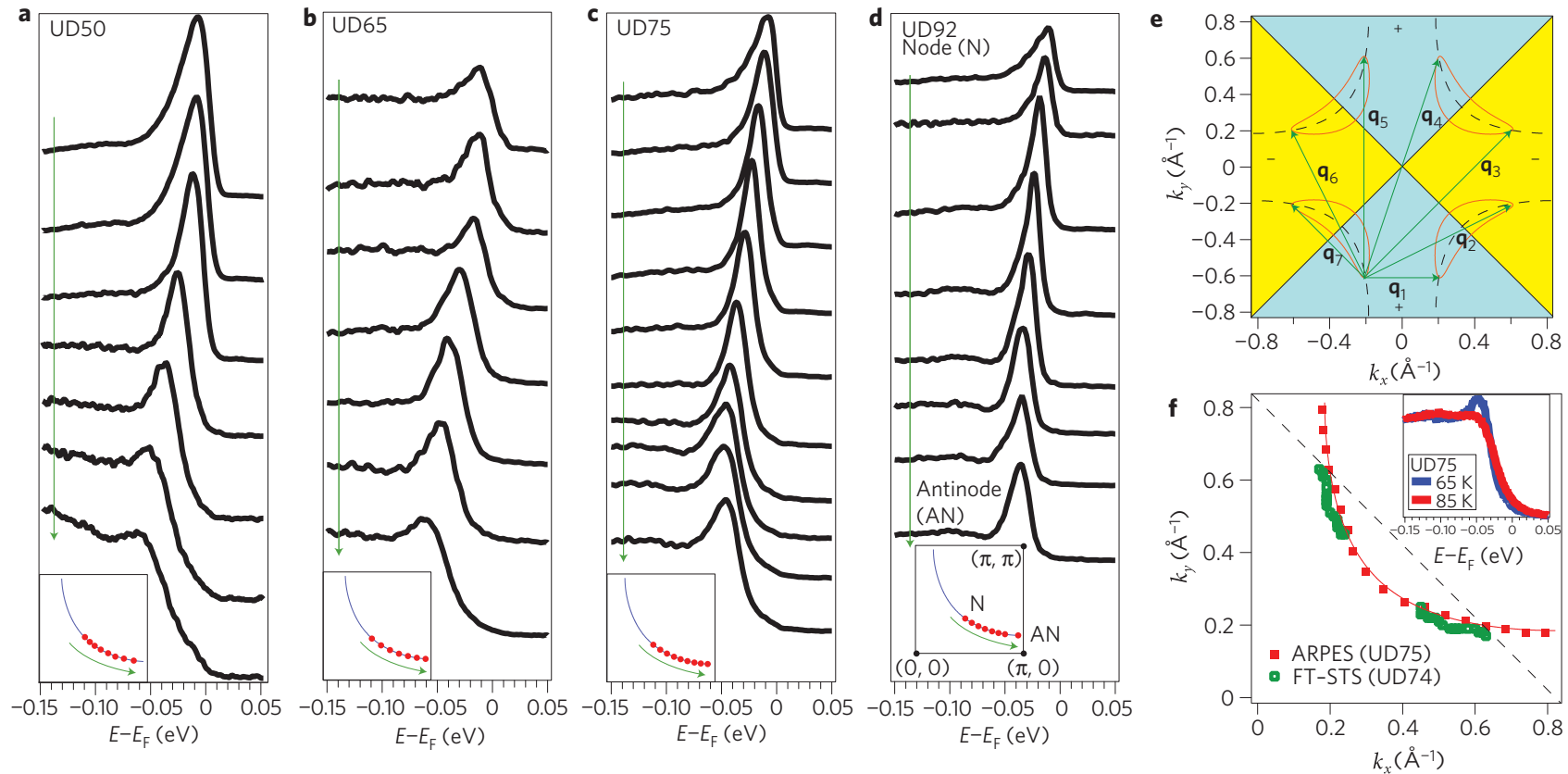

Figure 1 | Quasiparticles in ARPES data. a-d, EDCs at $\mathbf{k}_{\mathrm{F}}$ : node (top) to antinode (bottom). Insets: Fermi surface intersection for each cut. e, Octet model QPI wave vectors, $\mathbf{q}_{1}-\mathbf{q}_{7}$, connect the ends of CCEs (red solid lines) ${ }^{5}$ around the Fermi surface (dashed line), where blue (yellow) regions represent $\Delta(\mathbf{k})>0(\Delta(\mathbf{k})<0)$. f, Fourier-transform (FT) STS infers the Fermi surface by tracking dispersing QPI wave vectors, terminating at the antiferromagnetic zone boundary (dashed line) $)^{5}$. For a similar doping, ARPES detects quasiparticles extending to the antinode. Inset: UD75 EDCs at the antinode measured at $85 \mathrm{~K}$ and $65 \mathrm{~K}$, showing emergence of the quasiparticle peak near $T_{\mathrm{c}}$.
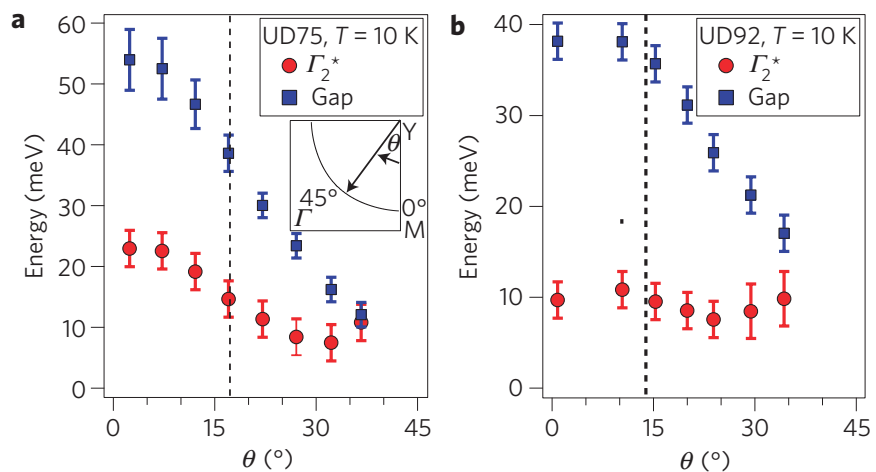

Figure 2 | Scattering-rate fits. a,b, Symmetrized EDCs at $\mathbf{k}_{\mathrm{F}}$ for UD75 (a) and UD92 (b) were fitted to an energy-dependent scattering rate, $\Gamma=\alpha \omega$, convolved with the experimental energy resolution. The characteristic scattering rate, $\Gamma_{2}^{*}=\alpha \Delta(\mathbf{k})$, is plotted as a function of Fermi surface angle along with the fitted $\Delta(\mathbf{k})$. Error bars for $\Gamma_{2}^{*}$ are estimated by the $3 \sigma$ confidence interval of the fitting and error bars for the gap are described in ref. 10. There is no evidence that the scattering rate diverges near the antiferromagnetic zone boundary for either doping. In addition, the peak width in the antinodal region is smaller than the gap, indicating that the peaks are quasiparticle-like.

the peaks are quasiparticle-like in this region of interest. For UD92, the quasiparticle width changes little from the node to the antinode, a result similar to earlier work on overdoped samples ${ }^{14}$. The UD75 scattering rate shows a stronger momentum dependence, but the overall variation is still only a factor of three. For both dopings, there is no anomaly in the scattering at the antiferromagnetic zone boundary, ruling out proposals for quasiparticle extinction that invoke a sudden increase in single-particle scattering there ${ }^{5}$.

What could plausibly explain the apparent contradiction between ARPES and Fourier-transform STS results? One intriguing observation is that not all QPI wave vectors in $\rho(\mathbf{q}, \omega)$ vanish across the antiferromagnetic zone boundary: $\mathbf{q}_{1,4,5}$ survive, whereas the others fall below the Fourier-transform STS noise floor ${ }^{5}$. Here, we study the effects of quasiparticle scattering from impurities on the basis of a weak-coupling approach ${ }^{15}$. Although this neglects the large, relevant, spatial inhomogeneity in the LDOS shown with STS (ref. 7), it places a simple focus on the differences between measurements, contrasting electron removal spectra in ARPES against two-particle QPI mechanisms in Fourier-transform STS.

On the basis of ARPES results, the electron propagators are described by the Nambu Green's function $\hat{G}$ in the superconducting state and the momentum-dependent $\hat{T}$ matrix determines the non-uniform part of $\rho(\mathbf{q}, \omega)$ (ref. 16):

$$
\delta \rho(q, \omega)=\frac{-1}{\pi} \operatorname{Im} \sum_{p}[\hat{G}(p, \omega) \hat{T}(p, p+q) \hat{G}(p+q, \omega)]_{11}
$$

where $\hat{G}(p, \omega)=1 /\left[\omega \hat{\tau}_{0}-\xi(p) \hat{\tau}_{3}-\Delta(p) \hat{\tau}_{1}\right]$ is written in terms of Pauli matrices $\hat{\tau}_{1,3}$, the unit matrix $\hat{\tau}_{0}$, the band structure $\xi(\mathbf{p})$ and the $d$-wave superconducting gap $\Delta(p)=\Delta_{0}[\cos$ $\left.\left(p_{x} a\right)-\cos \left(p_{y} a\right)\right] / 2$. Contributions to the $\hat{T}$ matrix can be classified according to how they modify electron parameters: conventional impurity scattering occurs in the $\hat{\tau}_{3}$ channel, whereas local superconducting gap modification occurs in the $\hat{\tau}_{1}$ channel.

A single impurity at site $(0,0)$ locally modifies hopping and the $d$-wave superconducting gap through an impurity contribution to the Hamiltonian ${ }^{17-19}: H=\sum_{r} \Psi_{r}^{\dagger}\left[\hat{\tau}_{3} \delta t(r)+\hat{t}_{1} \delta \Delta(r)\right] \Psi_{0}+$ h.c., where spin indices have been suppressed. The hopping and $d$-wave gap modulations, $\delta t(r)$ and $\delta \Delta(r)$, are proportional to $\delta(r-a x) \pm \delta(r-a y)+\delta(r+a x) \pm \delta(r+a y)$, where $a$ is the square lattice spacing and the upper (lower) sign is for hopping ( $d$-wave gap) modulation. Given this form of the impurity Hamiltonian, the Fourier transform, $\hat{T}(p, p+q)$, has a simple momentum-space form: $\hat{\tau}_{3,1}\left[\cos \left(p_{x}\right) \pm \cos \left(p_{y}\right)+\cos \left(p_{x}+q_{x}\right) \pm \cos \left(p_{y}+q_{y}\right)\right]$ for hopping and $d$-wave gap modulated scattering, respectively. In the $\hat{\tau}_{1}$ channel (gap modulation), scattering vanishes between points with opposite order-parameter phases. Thus, $\mathbf{q}_{2,3,6,7}$ vanish identically, 
a
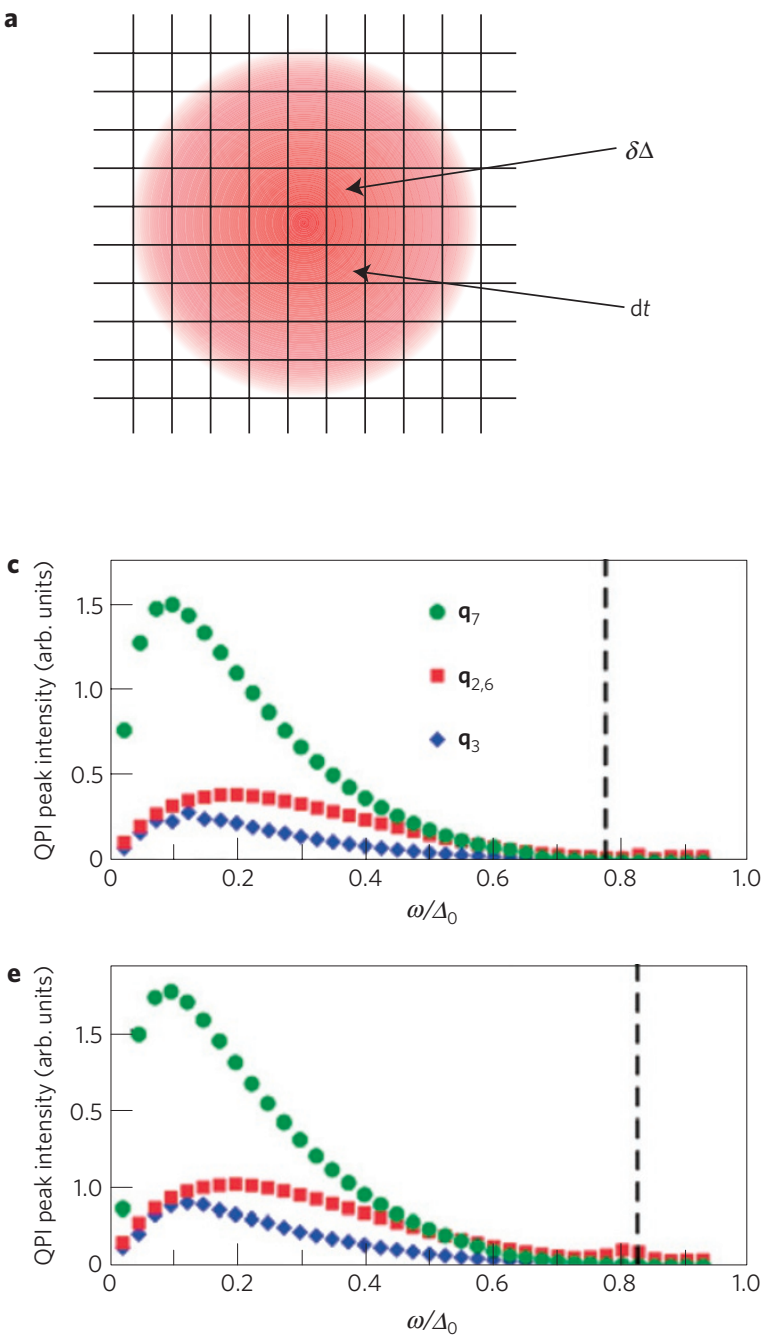

g

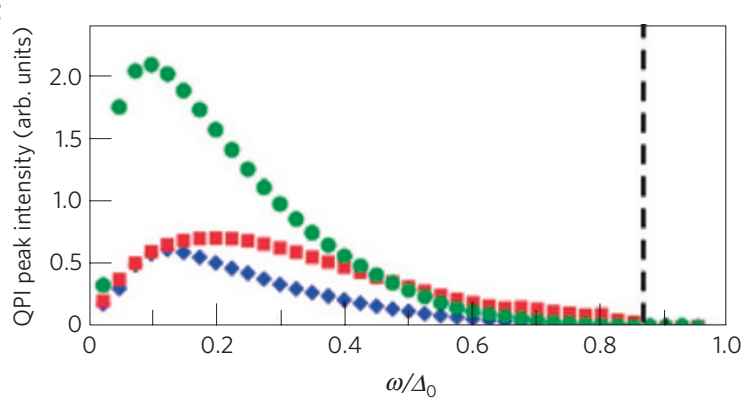

b

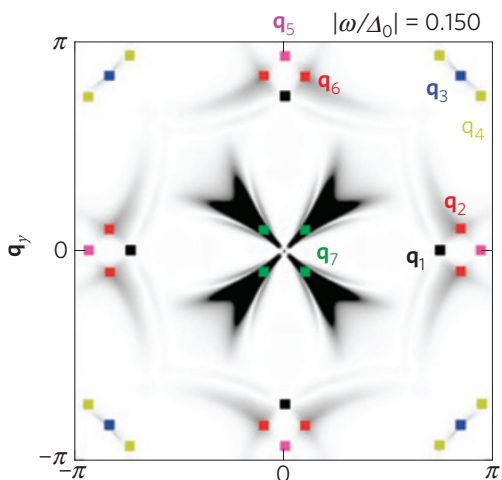

$\mathbf{q}_{x}$
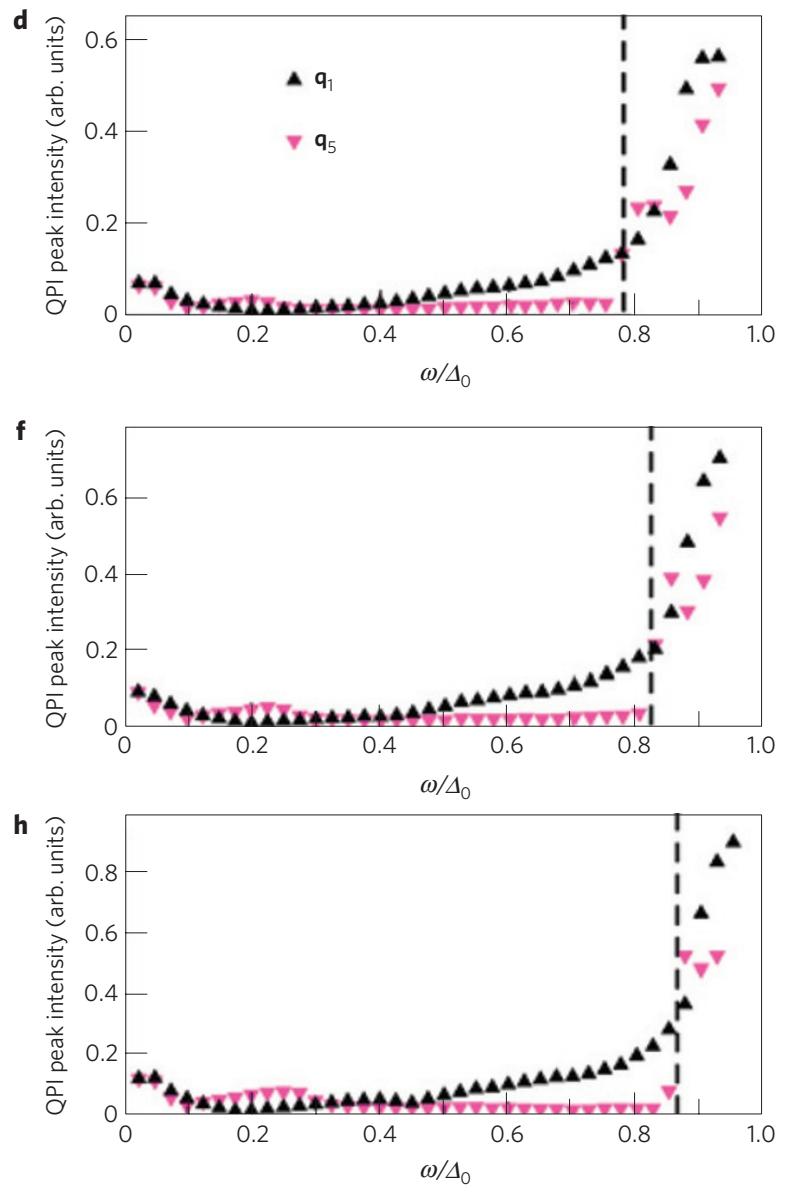

Figure 3 | Patch calculation. $\mathbf{a}$, Sketch of the patch used for calculations. $\mathbf{b}, Z$ map for $\left|\omega / \Delta_{0}\right|=0.15$. The QPI wave vectors $\mathbf{q}_{1}-\mathbf{q}_{7}$ are indicated. $\mathbf{c}-\mathbf{h}, \mathrm{QPI}$ peak intensities determined from the $Z$ map for $5 \%(\mathbf{c}, \mathbf{d}), 10 \%(\mathbf{e}, \mathbf{f})$ and $15 \%(\mathbf{g}, \mathbf{h})$ hole doping. $\mathbf{c}, \mathbf{e}$ and $\mathbf{g}$ show the intensity for wave vectors $\mathbf{q}_{7}, \mathbf{q}_{2,6}$ and $\mathbf{q}_{3}$. The dashed line in each panel indicates the energy where the tips of the CCEs cross the antiferromagnetic zone boundary, where the peak intensities associated with $\mathbf{q}_{2,3,6,7}$ vanish. $\mathbf{d}, \mathbf{f}$ and $\mathbf{h}$ show the intensities for wave vectors $\mathbf{q}_{1}$ and $\mathbf{q}_{5}$, which rise on approaching the antiferromagnetic zone boundary.

whereas $\mathbf{q}_{1,4,5}$ persist. In the $\hat{\tau}_{3}$ channel (hopping modulation), scattering between equivalent points leads to no such cancellation, but the momentum dependence of the $\hat{T}$ matrix implies a loss of intensity for $\mathbf{q}$ vectors connecting points $\mathbf{p}$ and $\mathbf{p}+\mathbf{q}$, which both lie on the antiferromagnetic zone boundary. Thus, the disappearance of QPI peaks associated with $\mathbf{q}_{2,3,6,7}$ at the antiferromagnetic zone boundary for the types of disorder considered here may be associated simply with the momentum dependence of the $\hat{T}$ matrix, rather than implying the 'extinction' of quasiparticles. This also may reconcile the observation that the QPI peaks vanish even at large dopings $(p \approx 0.19)$, a regime with diminished influence from the antiferromagnetic Mott insulating state.
The analysis presented above- a single impurity scatterer embedded in an infinite host-provides a simple view of the momentum dependence of the $\hat{T}$ matrix and a plausible explanation for the loss of QPI peak intensity at the antiferromagnetic zone boundary. However, it cannot generally describe the behaviour of Fourier-transform STS QPI peak intensity as a function of bias voltage or the implications associated with the Fourier transform of the $Z$ map, a ratio of the LDOS at positive and negative bias, rather than the LDOS itself. A better comparison with Fourier-transform STS results from considering the effects of an extended 'patch' impurity embedded in a finite-size, periodic host with the $\hat{T}$ matrix determined self-consistently. Finite-size effects are partially 
mitigated by smoothing the impurity with a Gaussian envelope, thus decreasing the influence of the impurity on electronic parameters further from the centre of the patch. In addition, the QPI intensity is determined from a Fourier transform of the $Z$ map, as done in Fourier-transform STS experiments ${ }^{4,5}$. More details of the calculation including the exact patch geometry and modulation parameters can be found in the Supplementary Information.

Figure $3 \mathrm{c}-\mathrm{h}$ shows results for $5 \%, 10 \%$ and $15 \%$ hole doping. The dashed line in each panel indicates the energy associated with the antiferromagnetic zone boundary. Note that the general trends agree with the initial analysis from a single impurity scatterer. Intensities for peaks $\mathbf{q}_{2,3,6,7}$, shown in Fig. 3c,e,g, initially rise on moving away from the nodal point and then begin to fall on approaching the antiferromagnetic zone boundary. The intensity for QPI peaks $\mathbf{q}_{1,5}$ is small initially and rises abruptly as the bias voltage approaches and crosses the energy associated with the antiferromagnetic zone boundary, both trends in agreement with the single impurity analysis. This behaviour is in qualitative agreement with the experimental intensity profiles as a function of energy for all QPI peaks ${ }^{5}$. Although there are general quantitative changes with band-structure parameters, impurity 'patch' size and shape and degree of modulation, the qualitative agreement with the single-impurity analysis and experimental findings remains robust.

Received 26 March 2009; accepted 28 July 2009; published online 30 August 2009

\section{References}

1. Damascelli, A., Hussain, Z. \& Shen, Z.-X. Angle-resolved photoemission studies of the cuprate superconductors. Rev. Mod. Phys. 75, 473-541 (2003).

2. Ding, H. et al. Coherent quasiparticle weight and its connection to high- $\mathrm{T}_{c}$ superconductivity from angle-resolved photoemission. Phys. Rev. Lett. 87, 227001 (2001).

3. McElroy, K. et al. Relating atomic-scale electronic phenomena to wave-like quasiparticle states in superconducting $\mathrm{Bi}_{2} \mathrm{Sr}_{2} \mathrm{CaCu}_{2} \mathrm{O}_{8+\delta}$. Nature 422, 592-596 (2003).

4. Hanaguri, T. et al. Quasiparticle interference and superconducting gap in $\mathrm{Ca}_{2-x} \mathrm{Na}_{x} \mathrm{CuO}_{2} \mathrm{Cl}_{2}$. Nature Phys. 3, 865-871 (2007).

5. Kohsaka, Y. et al. How Cooper pairs vanish approaching the Mott insulator in $\mathrm{Bi}_{2} \mathrm{Sr}_{2} \mathrm{CaCu}_{2} \mathrm{O}_{8+\delta}$. Nature 454, 1072-1078 (2008).

6. McElroy, K. et al. Coincidence of checkerboard charge order and antinodal state decoherence in strongly underdoped superconducting $\mathrm{Bi}_{2} \mathrm{Sr}_{2} \mathrm{CaCu}_{2} \mathrm{O}_{8+\delta}$. Phys. Rev. Lett. 94, 197005 (2005).

7. Lang, K. M. et al. Imaging the granular structure of high- $\mathrm{T}_{c}$ superconductivity in underdoped $\mathrm{Bi}_{2} \mathrm{Sr}_{2} \mathrm{CaCu}_{2} \mathrm{O}_{8+\delta}$. Nature 415, 412-416 (2002).
8. Feng, D. L. et al. Signature of superfluid density in the single-particle excitation spectrum of $\mathrm{Bi}_{2} \mathrm{Sr}_{2} \mathrm{CaCu}_{2} \mathrm{O}_{8+\delta}$. Science 289, 277-281 (2000).

9. Tanaka, K. et al. Distinct Fermi-momentum-dependent energy gaps in deeply underdoped Bi2212. Science 314, 1910-1913 (2006).

10. Lee, W. S. et al. Abrupt onset of a second energy gap at the superconducting transition of underdoped Bi2212. Nature 450, 81-84 (2007).

11. Sato, T. et al. Low energy excitation and scaling in $\mathrm{Bi}_{2} \mathrm{Sr}_{2} \mathrm{Ca}_{n-1} \mathrm{Cu}_{n} \mathrm{O}_{2 n+4}$ ( $n=1-3)$ : Angle-resolved photoemission spectroscopy. Phys. Rev. Lett. 89, 067005 (2002).

12. Bansil, A., Lindroos, M., Sahrakorpi, S. \& Markiewicz, R. S. Influence of the third dimension of quasi-two-dimensional cuprate superconductors on angle-resolved photoemission spectra. Phys. Rev. B 71, 012503 (2005).

13. Alldredge, J. W. et al. Evolution of the electronic excitation spectrum with strongly diminishing hole density in superconducting $\mathrm{Bi}_{2} \mathrm{Sr}_{2} \mathrm{CaCu}_{2} \mathrm{O}_{8+\delta}$. Nature Phys. 4, 319-326 (2008).

14. Bogdanov, P. V. et al. Anomalous momentum dependence of the quasiparticle scattering rate in overdoped $\mathrm{Bi}_{2} \mathrm{Sr}_{2} \mathrm{CaCu}_{2} \mathrm{O}_{8}$. Phys. Rev. Lett. 89, 167002 (2002).

15. Fang, A. C. et al. Gap-inhomogeneity-induced electronic states in superconducting $\mathrm{Bi}_{2} \mathrm{Sr}_{2} \mathrm{CaCu}_{2} \mathrm{O}_{8+\delta}$. Phys. Rev. Lett. 96, 017007 (2006).

16. Kampf, A. P. \& Devereaux, T. P. Extended impurity potential in a $d_{x} 2-{ }_{y} 2$ superconductor. Phys. Rev. B 56, 2360-2363 (1997).

17. Zhu, J. X. Dopant-induced local pairing inhomogeneity in $\mathrm{Bi}_{2} \mathrm{Sr}_{2} \mathrm{CaCu}_{2} \mathrm{O}_{8+\delta}$. Preprint at <http://arxiv.org/abs/cond-mat/0508646> (2005).

18. Nunner, T. S., Chen, W., Andersen, B. M., Melikyan, A. \& Hirschfeld, P. J. Fourier transform spectroscopy of $d$-wave quasiparticles in the presence of atomic scale pairing disorder. Phys. Rev. B 73, 104511 (2006).

19. Johnston, S., Vernay, F. \& Devereaux, T. P. Impact of an oxygen dopant in $\mathrm{Bi}_{2} \mathrm{Sr}_{2} \mathrm{CaCu}_{2} \mathrm{O}_{8+\delta}$. Europhys. Lett. 86, 37007 (2009).

\section{Acknowledgements}

We thank N. Nagaosa and J. Zaanen for helpful discussions. SSRL is operated by the US Department of Energy, Office of Basic Energy Science, Division of Chemical Science and Material Science. This work is supported by the US Department of Energy, Office of Science, Division of Materials Science, with contracts DE-AC02-76SF00515, DE-FG03-01ER45929-A001 and NSF grant DMR-0604701.

\section{Author contributions}

I.M.V. analysed ARPES data and wrote the portions of the manuscript and Supplementary Information relevant to ARPES results. W.-S.L. carried out ARPES experiments on samples grown by T.S. and K.T. carried out experiments on samples grown by T.F. B.M. and E.A.N. wrote and modified the computer codes and carried out the computational simulations. T.P.D., B.M. and E.A.N. wrote and edited those portions of the main text and Supplementary Information relevant to the simulations. T.P.D. and Z.-X.S. are responsible for project direction, planning and infrastructure.

\section{Additional information}

Supplementary information accompanies this paper on www.nature.com/naturephysics. Reprints and permissions information is available online at http://npg.nature.com/ reprintsandpermissions. Correspondence and requests for materials should be addressed to T.P.D. 